Article

\title{
Hydromagnetic Dissipative and Radiative Graphene Maxwell Nanofluid Flow Past a Stretched Sheet-Numerical and Statistical Analysis
}

\author{
Syed M. Hussain ${ }^{1, *}$, Rohit Sharma ${ }^{2}$, Manas R. Mishra ${ }^{3}$ and Sattam S. Alrashidy ${ }^{1}$ \\ 1 Department of Mathematics, Faculty of Science, Islamic University of Madinah, Madinah 42351, \\ Saudi Arabia; sattamjr@gmail.com \\ 2 Department of Mathematics, GITAM Bengaluru, Karnataka 562163, India; rohit.iitg08@gmail.com \\ 3 Department of Mathematics, School of Science, OP Jindal University, Raigarh 496109, India; \\ manas.mishra@opju.ac.in \\ * Correspondence: syed.hussain@iu.edu.sa or hussain.modassir@yahoo.com; Tel.: +96-65-5990-9721
}

Received: 17 September 2020; Accepted: 21 October 2020; Published: 2 November 2020

\begin{abstract}
The key objective of this analysis is to examine the flow of hydromagnetic dissipative and radiative graphene Maxwell nanofluid over a linearly stretched sheet considering momentum and thermal slip conditions. The appropriate similarity variables are chosen to transform highly nonlinear partial differential equations (PDE) of mathematical model in the form of nonlinear ordinary differential equations (ODE). Further, these transformed equations are numerically solved by making use of Runge-Kutta-Fehlberg algorithm along with the shooting scheme. The significance of pertinent physical parameters on the flow of graphene Maxwell nanofluid velocity and temperature are enumerated via different graphs whereas skin friction coefficients and Nusselt numbers are illustrated in numeric data form and are reported in different tables. In addition, a statistical approach is used for multiple quadratic regression analysis on the numerical figures of wall velocity gradient and local Nusselt number to demonstrate the relationship amongst heat transfer rate and physical parameters. Our results reveal that the magnetic field, unsteadiness, inclination angle of magnetic field and porosity parameters boost the graphene Maxwell nanofluid velocity while Maxwell parameter has a reversal impact on it. Finally, we have compared our numerical results with those of earlier published articles under the restricted conditions to validate our solution. The comparison of results shows an excellent conformity among the results.
\end{abstract}

Keywords: graphene maxwell nanofluid; magnetic field; thermal radiation; joule dissipation; viscous dissipation

\section{Introduction}

In recent years, the nanofluids problems are attracting noteworthy attention of researchers owing to promising significance in industry and public endeavour as nanofluids possess the noble heat transfer characteristics to boost the conventional fluid's performance. The nanofluid foremost termed by Choi and Eastman [1] is a stable colloidal assortment of extremely fine non-metallic/metallic particles (up to $50 \mathrm{~nm}$ in radius) in conventional fluids like oil, water, some lubricants, and ethylene glycol, etc. Addition of extremely fine non-metallic/metallic particles in the conventional fluid influences the fluid characteristics like heat transfer rate and thermal conductivity [2]. Nanofluids are enormously useful in broad prospects including energy conversion, microsystem's cooling (due to change in heat capacity of base fluid by mixing nano-sized metal oxides/metal particles), nanomedical industry, sensor and optical devices $[3,4]$. Nowadays, graphene nanoparticles are acquiring influential scientific and technological attention with probable applications such as in batteries, miniaturized, solar or 
fuel cells, biomedical sensors and fabrication of supercapacitors. Owing to fast mobility of electrons, high thermal conductivity, stability, cell growth capability, expanded surface area and biocompatibility certainties the graphene nanoparticles possess the novel material, physical, electrical and chemical characteristics [5]. Therefore, it has extensive applications including but not restricted to electronics, energy sector, sensing outlets, medical sciences, etc. [6]. Upadhya et al. [7] performed the theoretical investigation of magneto-Carreau fluid by adding the graphene and dust particles into it and observed that mixture of ethylene glycol and graphene nanoparticles are noteworthy in improving the heat transport phenomena.

In general, the dispersion of very fine nanoparticles into Newtonian conventional fluid changes the characteristics of fluid to non-Newtonian. Numerous fluids used in various engineering and industrial processes are non-Newtonian fluids in characteristics, for instance, molten plastics, polymers, nuclear fuel slurries, pulps, mercury amalgams, liquid metals, lubrication by heavy oil, etc. Owing to complex characteristics of these fluids one cannot find a single constitutive equation that reveals all characteristics of such non-Newtonian fluids. However, several complex constitutive models for the non-Newtonian fluids are anticipated by a number of researchers, namely, Serdar and Dokuz [8], Haroun [9], Sajid et al. [10] and Hayat et al. [11], etc. The proposed models are concerned with the second, third and fourth grade fluids which can estimate the impact of elasticity while these models are shear independent and unable to envisage the significance of shear relaxation [12]. Moreover, Maxwell model is proposed for the rate kind fluids which can estimate shear stress relaxation and consequently developed a popular model among the researchers. These models can also depict the significance of shear-dependent viscosity of boundary layer problems. Following this, many researchers, namely, Fetecau and Fetecau [13], Wang and Hayat [14], Fetecau [15], Hayat et al. [16] and Heyhyat and Khabazi [17] reported their investigations on Maxwell fluid flow problems considering different geometrical configurations to analyze the significance of different germane parameters on the flow field.

The innovative characteristics of thermal radiation for surface heat transfer cannot be ignored while considering the industrial processes like electrical power generation, designing of furnace, production of glass, missiles devices, solar power technology, etc., which occur at high temperature. In the present industrial scenario due to decrease of conventional energy resources the attention has been focused on sustainable and renewable energy sources. The main fountain of renewable energy is solar energy and thermal radiation acts a noteworthy part in transforming the solar energy to the appropriate form for various applications in industry. Following this, Wang et al. [18] investigated the significance of thermal radiation on non-Newtonian hydromagnetic stagnation point flow considering Ohmic heating into account and observed that radiation parameter enhances the heat transfer rate of fluid. Further, an innovative thermal conductivity model considering dynamic and static approach was proposed by Nayak et al. [19] to illustrate the thermal radiation impact on Darcy-Forchheimer nanofluid flow past a rotating disk. Recently, Khan and Alzahrani [20] explored the impacts of nonlinear thermal radiation, binary chemical reaction and activation energy on stagnation point flow of hydromagnetic Walter-B nanofluid and noticed that nanofluid temperature gets augmented owing to enhancement in thermophoresis diffusion coefficient, thermal Biot number and activation energy parameter. Some recent distinguished research studies mentioning the novel characteristics of thermal radiation on different types of flow fields can be found in references [21-28].

The viscous dissipation impact is not considered in above described studies as the same is supposed to be low but its relevance in food processing, instrumentations, lubrications, polymer manufacturing, etc., is noteworthy as it enhances the characteristics of temperature distribution and consequently induce the heat transfer rate. Some important studies dealing with viscous dissipative flow problems induced by stretched sheets are reported in literature [29-32]. Further, Joule dissipation shows the characteristics alike to volumetric heat source in magnetohydrodynamic fluid flows and the collective influence of Joule and viscous dissipations are imperative in context of heat-treated materials. Following this, several researchers including Daniel et al. [33], Seth and Singh [34] and Seth et al. [35] modelled their problems considering the impacts of Joule and viscous dissipations. Abbas et al. [36] studied the 
entropy optimized, dissipative and hydromagnetic Darcy-Forchheimer flow of propylene glycol based nanofluid past a stretched sheet and numerical results are computed using Built-in-Shooting Technique. Wang et al. [37] worked on irreversibility characteristics in hydromagnetic entropy optimized nanofluid flow by variable thick surface subject to viscous dissipation and Joule heating. Flow behaviour of entropy optimized hydromagnetic micropolar Casson-nanofluid subject to Joule-heating, viscous dissipation and nonlinear thermal radiation was scrutinized by Nayak et al. [38]. Recently, Ibrahim and Khan [39] explored the impacts of viscous dissipation on the mixed convective flow of MWCNT and SWCNT water driven nanofluids in a porous medium past a stretched sheet.

A meticulous review of research papers reported in literature reveals that the majority of researchers have considered the no-slip conditions in their investigations and have ignored the slip conditions. But, in many physical problems such as low-pressure flows, micro/nano-scale flows, flow over coated surfaces, etc. Navier's partial slip took place. Owing to this reason, Mukhopadhyay and Gorla [40], Singh and Makinde [41] and Seth et al. [42,43] studied boundary layer hydromagnetic fluid flow problems taking partial slip conditions into account. Some novel published articles dealing with the latest advancements in aforesaid fields are listed in references [44-50].

The present study is mainly concentrated to unfold the significance of thermal radiation, Joule and viscous dissipations on the flow of graphene Maxwell nanofluid past a linearly horizontal stretched sheet under the momentum and thermal slip conditions. The highly complex prevailing equation of the problem is numerically solved employing Runge-Kutta-Fehlberg algorithm along with the shooting scheme. Further, a statistical approach is followed for multiple regression estimation analysis on numerical figures of wall velocity gradient and local Nusselt number. A careful review of research papers reported in literature reveals that none of the authors has attempted this problem earlier although the thoughts, methodology and results explained in this paper can be useful in electronics, energy sector, sensing outlets and medical sciences, etc.

\section{Formulation of Problem in Mathematical Form}

In this problem, we have considered flow of an incompressible, optically dense radiative, two-dimensional stream line and electrically conductive non-Newtonian Maxwell graphene nanofluid over a linearly stretched sheet as displayed in Figure 1. The nanofluid is prepared by dispersing graphene nanoparticles into ethylene glycol. It is assumed that the graphene nanoparticles are having unvarying shape and size. In addition, ethylene glycol and graphene nanoparticles are presumed in thermal equilibrium state as well no-slip happens between them. The thermophysical properties of ethylene glycol and graphene nanoparticles are provided in Table 1.

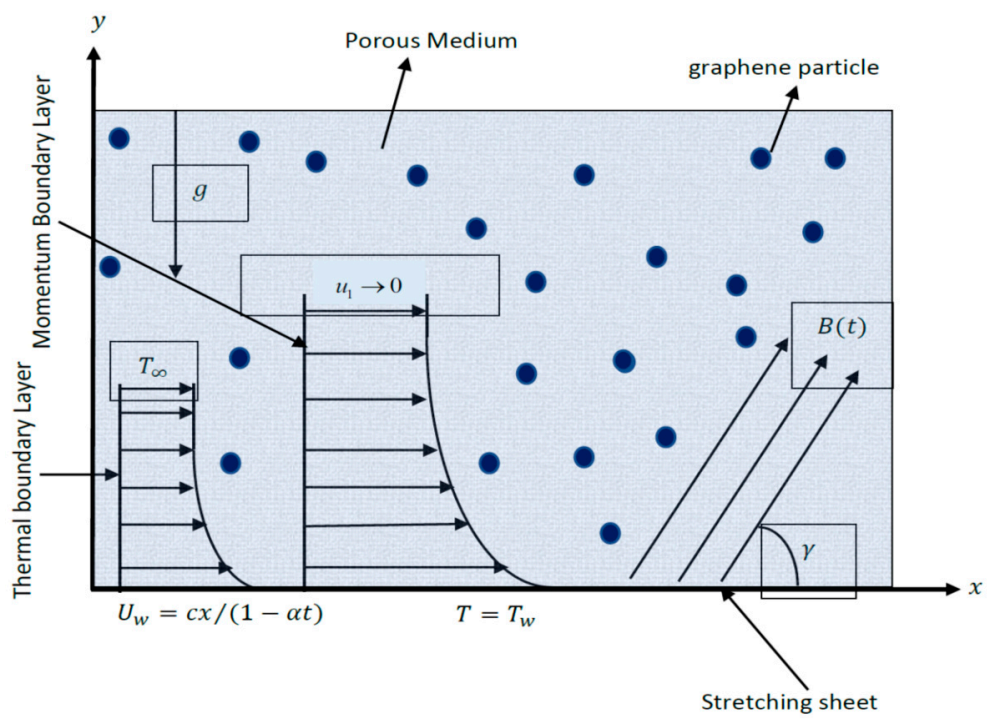

Figure 1. Physical illustration of the problem. 
Table 1. Thermophysical properties of Ethelene glycol and graphene nanoparticles [51,52].

\begin{tabular}{ccccc}
\hline Thermo-Physical Properties & $\mathrm{K}(\mathrm{W} / \mathbf{m K})$ & $\mathrm{c}_{p}(\mathrm{~J} / \mathbf{k g K})$ & $\rho\left(\mathbf{k g} / \mathbf{m}^{3}\right)$ & $\sigma(\mathrm{S} / \mathbf{m})$ \\
\hline Ethelene glycol & 0.252 & 2415 & 1114 & $5.5 \times 10-6$ \\
Graphene & 2500 & 2100 & 2250 & $1 \times 107$ \\
\hline
\end{tabular}

The two-dimensional Cartesian coordinate system is chosen to portray the flow geometry of nanofluid. The linear stretching sheet is aligned in $x$-direction while $y$-axis is upright to the stretching sheet. The nanofluid is induced over a stretching sheet owing to the unsteady magnetic field $B(t)=B_{0}(1-\alpha t)^{-1 / 2}$ exerted along $y$ - axis and flow-field is confined in the domain $y>0$. Further, following Cramer and Pai [53] the tempted magnetic field influence is presumed to be ignorable in comparison to exerted magnetic field. The optically thick radiative Maxwell nanofluid is considered, therefore, Rosseland approximation is taken into account to do the mathematical model of the problem [23]. Moreover, the considered sheet is anticipated to be shrunk owing to two opposite but equal forces with time dependent velocity $U_{w}(x, t)=c x(1-\alpha t)^{-1}$, where $c$ and $(1-\alpha t)^{-1}$ (here $\left.\alpha t<1\right)$ are respectively initial and notable stretching rate. The wall temperature of nanofluid is considered $T_{w}$ while the temperature outside the boundary regime is known as free stream and is denoted by $T_{\infty}$.

Owing to assumption made in this problem, the prevailing mathematical equations of momentum and energy for graphene Maxwell nanofluid are reduced to (for descriptions, see Mukhopadhyay [54]):

$$
\begin{gathered}
\frac{\partial u_{1}}{\partial x}+\frac{\partial u_{2}}{\partial y}=0, \\
\frac{\partial u_{1}}{\partial t}+u_{1} \frac{\partial u_{1}}{\partial x}+u_{2} \frac{\partial u_{1}}{\partial y}=\frac{\mu_{n f}}{\rho_{n f}} \frac{\partial^{2} u_{1}}{\partial y^{2}}-\left\{\frac{\mu_{n f}}{\rho_{n f} \mathrm{~K}} u_{1}+\frac{\sigma_{n f} B^{2}(t) u_{1}}{\rho_{n f}} \sin ^{2}(\gamma)\right\}- \\
\lambda\left(u_{1}^{2} \frac{\partial^{2} u_{1}}{\partial x^{2}}+u_{2}{ }^{2} \frac{\partial^{2} u_{1}}{\partial y^{2}}+2 u_{1} u_{2} \frac{\partial^{2} u_{1}}{\partial x \partial y}\right), \\
\frac{\partial T}{\partial t}+u_{1} \frac{\partial T}{\partial x}+u_{2} \frac{\partial T}{\partial y}=\frac{k_{n f}}{\left(\rho c_{p}\right)_{n f}}\left(\frac{\partial^{2} T}{\partial y^{2}}\right)+\frac{\sigma_{n f} B^{2}(t) u_{1}{ }^{2}}{\left(\rho c_{p}\right)_{n f}} \sin ^{2}(\gamma)+\frac{\mu_{n f}}{\rho_{n f}}\left(\frac{\partial u_{1}}{\partial y}\right)^{2}-\frac{1}{\left(\rho c_{p}\right)_{n f}} \frac{\partial q_{r}}{\partial y} .
\end{gathered}
$$

The allied conditions for the physical problem are:

$$
\begin{gathered}
u_{1}(x, 0)=U_{w}(x, 0)+s \frac{\mu_{n f}}{\rho_{n f}} \frac{\partial u_{1}}{\partial y}, u_{2}=0, T(x, 0)=T_{w}+D \frac{\partial T}{\partial y}, \\
u_{1} \rightarrow 0, T \rightarrow T_{\infty} \text { when } y \rightarrow \infty .
\end{gathered}
$$

In Equations (1)-(5), notations $u_{1}$ and $u_{2}$ represent the graphene Maxwell nanofluid velocities along the $x$-direction (primary velocity) and $y$-direction (secondary velocity) respectively. $T, \mu_{n f}, \sigma_{n f}, k_{n f}, \rho_{n f}$ and $\left(\rho c_{p}\right)_{n f}$ are respectively used to represent the temperature, dynamic viscosity, electrical conductivity, thermal conductivity, density and specific heat capacitance of graphene Maxwell nanofluid. The relation $\lambda=\lambda_{0}(1-\alpha t)$ represents the thermal relaxation, wherein $\lambda_{0}$ reflects the initial relaxation rate while $K=K_{0}(1-\alpha t)$ is the porosity parameter. The angle of inclination of exerted magnetic field in anti-clockwise sense from stretching surface is indicated by $\gamma$. The thermal radiative heat flux of graphene Maxwell nanofluid is mentioned by $q_{r}$ while slipping factor is represented by relation $s=s_{0} \sqrt{1-\alpha t}$ where $s_{0}$ is used to indicate the slip parameter at $t=0$. The relation $D=D_{0}(1-\alpha t)$ presents the slipping factor of fluid temperature where $D_{0}$ is the thermal slip parameter at $t=0$.

By making use of Rosseland approximation (for explanation, see Brewster [55]), for an optically thick radiative graphene Maxwell nanofluid, thermal radiative heat flux $q_{r}$ takes the following form:

$$
q_{r}+\frac{4 \sigma^{*}}{3 k^{*}} \frac{\partial T^{4}}{\partial y}=0
$$


Here, $\sigma^{*}$ is Stefan-Botzmann constant and $k^{*}$ signifies absorption coefficient.

\section{Numerical Solution of Problem}

The aforesaid physical problem consists of a set of coupled partial differential equations. So, to analyze the solution of these equations, foremost, we have introduced the stream functions $\psi$ along with the similarity variable $\eta$ to transform Equations (2)-(6) in the form of ordinary differential equations as under:

$$
\begin{gathered}
u_{1}=\frac{\partial \psi}{\partial y}, u_{2}=-\frac{\partial \psi}{\partial x} \\
\psi=\sqrt{\frac{c v_{f}}{1-\alpha t}} x f(\eta), \theta=\frac{T-T_{\infty}}{T_{w}-T_{\infty}} \text { and } \eta=\sqrt{\frac{c}{(1-\alpha t) v_{f}} y .}
\end{gathered}
$$

By making use of above relations (7)-(8), the prevailing set of mathematical Equations (2)-(6) is reduced to below mentioned dimensionless forms:

$$
\begin{gathered}
\phi_{a} \frac{f^{\prime \prime \prime}}{\phi_{b}}+\beta\left(2 f f^{\prime} f^{\prime \prime}-f^{2} f^{\prime \prime \prime}\right)-A\left(f^{\prime}+\frac{\eta}{2} f^{\prime \prime}\right)+\left(f f^{\prime \prime}-f^{\prime 2}\right)+\left\{K_{1}+\frac{\phi_{e}}{\phi_{b}} M \sin ^{2}(\gamma)\right\} f^{\prime}=0, \\
\left(\phi_{d}+N r \operatorname{Pr}\right) \theta^{\prime \prime}+\operatorname{Pr} \phi_{c}\left(\frac{\phi_{e}}{\phi_{c}} M E c \sin ^{2}(\gamma) f^{\prime 2}-\frac{A \eta}{2} \theta^{\prime}+f \theta^{\prime}+E c \frac{\phi_{a}}{\phi_{c}} f^{2}\right)=0 .
\end{gathered}
$$

Associated conditions are:

$$
\begin{gathered}
A_{1} f^{\prime \prime}(0)+f^{\prime}(0)=1, f(0)=0, \varepsilon \theta^{\prime}(0)+\theta(0)=1, \\
\text { as } \eta \rightarrow \infty, f^{\prime}(\eta) \rightarrow 0, \theta(\eta) \rightarrow 0,
\end{gathered}
$$

where

$$
\left.\begin{array}{c}
\phi_{a}=\frac{\mu_{n f}}{\mu_{f}}=\frac{1}{(1-\phi)^{2.5}}, \phi_{b}=\frac{\rho_{n f}}{\rho_{f}}=(1-\phi)+\frac{\rho_{s}}{\rho_{f}} \phi, \phi_{c}=\frac{\left(\rho c_{p}\right)_{n f}}{\left(\rho c_{p}\right)_{f}}=(1-\phi)+\phi \frac{\left(\rho c_{p}\right)_{s}}{\left(\rho c_{p}\right)_{f}}, \\
\phi_{d}=\frac{k_{n f}}{k_{f}}=\frac{k_{s}-2 \phi\left(k_{f}-k_{s}\right)+2 k_{f}}{k_{s}+\phi\left(k_{f}-k_{s}\right)+2 k_{f}}, \phi_{e}=\frac{\sigma_{n f}}{\sigma_{f}}=\left(1+\frac{3\left(\frac{\sigma_{s}}{\sigma_{f}}-1\right) \phi}{\left(\frac{\sigma_{s}}{\sigma_{f}}+2\right)-\left(\frac{\sigma_{s}}{\sigma_{f}}-1\right) \phi}\right) .
\end{array}\right\}
$$

In above expressions, $\rho_{f}$ is the base fluid's density, $\mu_{f}$ is the viscosity of Maxwell fluid (base fluid), $k_{s}$ represents thermal conductivity of graphene particles, $k_{f}$ represents the thermal conductivity of Maxwell fluid (base fluid), $\sigma_{s}$ represents the electrical conductivity of graphene nanoparticle, $\sigma_{f}$ represents the electrical conductivity of ethylene glycol, $A=\frac{\alpha}{c}$ is unsteadiness parameter, $\phi$ embodies volume fraction of nanoparticle in nanofluid, $\beta=c \lambda_{0}$ denotes Maxwell parameter, $M=\frac{\sigma_{f} B_{0}^{2}}{c \rho_{f}}$ presents magnetic parameter, $K_{1}=\frac{v_{f}}{c K}$ denotes the porous medium parameter, $\operatorname{Pr}=\frac{v_{f}}{\alpha_{f}}$ indicates the thermal diffusivity parameter, $N r=\frac{16}{3} \frac{\sigma^{*} T_{\infty}^{3}}{k^{*} v_{f}\left(\rho c_{p}\right)_{f}}$ implies radiation parameter, $E c=\frac{U_{w}^{2}}{\left(c_{p}\right)_{f}\left(T_{w}-T_{\infty}\right)}$ is local Eckert number demonstrations, $A_{1}=s_{0} \sqrt{\frac{c}{v_{f}}}$ denotes velocity slip parameter and $\varepsilon=D_{0} \sqrt{\frac{c}{v_{f}}}$ represents thermal slip parameter.

From engineering applications view point, the expressions for $S_{f}$ characterizes skin friction coefficient and local Nusselt number $N u_{x}$ are defined as:

$S_{f}=\frac{\tau_{w}}{\rho_{f} u_{w}^{2}}, N u_{x}=\frac{x q_{w}}{k_{f}\left(T_{w}-T_{\infty}\right)}$, where $\tau_{w}$ and $q_{w}$ are respectively presented as:

$$
\tau_{w}=\mu_{n f} \frac{\partial}{\partial y}\left(u_{1}+\lambda u_{2} \frac{\partial u_{1}}{\partial y}\right)_{y=0}, q_{w}=-k_{n f}\left(1+\frac{16}{3} \frac{\sigma^{*} T_{\infty}^{3}}{k^{*} v_{f}\left(\rho c_{p}\right)_{f}}\right)\left(\frac{\partial T}{\partial y}\right)_{y=0}
$$


The dimensionless form of skin friction coefficient and Nusselt number are expressed as:

$$
\left.\begin{array}{c}
S_{f} \sqrt{R e_{x}}=(1-f)^{-2.5}\left\{f^{\prime \prime}(0)-\lambda_{1} f(0) f^{\prime \prime \prime}(0)+\lambda_{1} f^{\prime}(0) f^{\prime \prime}(0)\right\}, \\
N u=-(1+N r) f_{d} \sqrt{R e_{x}} \theta^{\prime}(0),
\end{array}\right\}
$$

where $\lambda_{1}=\lambda_{0} c$ is the fluid relaxation parameter and $R e_{x}=\frac{x U_{w}(x, t)}{v_{f}}$ is the local Reynolds number.

\subsection{Implementation of Numerical Method}

Owing to complexity and highly nonlinear nature of the equations mentioned in Section 3, the equations cannot be solved analytically. Therefore, we have analyzed the numerical solution by making use of one of the reliable numerical method i.e., Runge-Kutta-Fehlberg method in combination with the shooting technique. Foremost, we have transformed the dimensionless form of Equations (9) and (10) into the set of five first order differential equations. Furthermore, algorithm of Runge-Kutta-Fehlberg is instigated to solve these differential equations where the initial functional values i.e., $f^{\prime \prime}(0)$ and $\theta^{\prime}(0)$ are obtained by means of shooting technique. The value of the similarity variable $\eta$ is chosen as $\eta=7$ aimed at infinity boundary conditions whereas the step size is considered as 0.001 during the numerical computation. To achieve precise results the tolerance error is taken as $10^{-6}$. The complete method is repeated till the anticipated correctness in the results is attained.

\subsection{Validation of Numerical Solution}

In this subsection, we have validated the obtained numerical solutions and correctness of implemented algorithm of numerical method as mentioned in Section 3.1 by comparing our results of $-f^{\prime \prime}(0)$ for varying unsteadiness parameter $A$ and $P r=0.1$ with those of Elbashbeshy and Bzid [56] and is shown in Table 2. An excellent conformity among both results can be seen in the comparison which validates the correctness of obtained numerical solution and implemented algorithm of numerical method.

Table 2. Comparison of $-f^{\prime \prime}(0)$ with Elbashbeshy and Bzid [56] when $\beta=M=K_{1}=E c=A 1=\varepsilon=0$.

\begin{tabular}{ccc}
\hline \multirow{2}{*}{$\boldsymbol{P r} / \boldsymbol{A}$} & \multicolumn{2}{c}{$\mathbf{0 . 1}$} \\
\cline { 2 - 3 } & Elbashbeshy and Bzid [56] & Present Result \\
\hline 0.8 & 1.3321 & 1.3333 \\
1.2 & 1.4691 & 1.4684 \\
2 & 1.7087 & 1.7090 \\
\hline
\end{tabular}

\section{Results and Discussion}

In this section, the obtained numerical solution by means of the method reported in Section 3 is presented to illustrate the significance of influencing physical parameters such as magnetic parameter $(M)$, porosity parameter $\left(K_{1}\right)$, inclination angle of magnetic field $(\gamma)$, Maxwell parameter $(\beta)$, unsteadiness parameter $(A)$, thermal radiation parameter $(N r)$, Eckert number $(E c)$ and thermal slip parameter $(\varepsilon)$ on the flow field. Throughout the numerical computation, values of various parameters are chosen as: radiation parameter $N r=0.2$, Prandtl number $P r=5$, unsteadiness parameter $A=0.1$, thermal slip parameter $\varepsilon=0.1$, Eckert number $E c=0.1$, angle of inclination of magnetic field $\gamma=\pi / 3$, magnetic parameter $M=1$, Maxwell parameter $\beta=0.5$ and porosity parameter $K_{1}=0.1$. These values remain unchanged throughout the analysis while varying values of parameters are specified in respective figures. Figures 2-6 demonstrate the impact of magnetic field, porous medium, inclination angle of magnetic field, Maxwell and unsteadiness parameters on the velocity of graphene Maxwell nanofluid whereas Figures 7-10 describe the significance of thermal radiation, inclination angle of magnetic field, thermal slip parameter and viscous dissipation on the temperature of graphene Maxwell nanofluid. In depicted figures, solid lines indicate the impact of pertinent flow parameters 
under no-slip condition while dashed lines denote the impact under slip condition. Figure 2 exhibits that graphene Maxwell fluid velocity decreases because of the increase in magnetic field parameter $(M)$ under both slip $(A=0.3)$ and no-slip $(A=0)$ velocity conditions. This type of behaviour happens due to resistive Lorentz force, which gets enhanced owing to the increase of magnetic field strength. Figure 3 portrays the porosity parameter $\left(K_{1}\right)$ effect on nanofluid velocity. It is seen that nanofluid fluid velocity gets reduced due to upsurge in porosity parameter. Physically it is interpreted as the increasing values of $K_{1}$ results the enhancement in resistance of fluid and as a consequence the nanofluid velocity gets reduced. It is clearly manifest from Figure 4 that enhancement in angle of exerted magnetic field $(\gamma)$ from stretching sheets results in slowing down nanofluid fluid velocity. This infers that, inclination angle of exerted magnetic tends to retard the graphene Maxwell nanofluid velocity and when the magnetic field is imposed in the transverse direction to the stretched sheet then the strength of resistive force is optimum. This optimum resistive force termed as Lorentz force which suppresses the fluid motion. So, in practical situations if it is required to augment the motion of fluid velocity then instead of exerting the magnetic field in transverse direction it may be exerted along the flow direction. Therefore, the graphene Maxwell nanofluid can be augmented or retarded by altering the inclination angle of exerted magnetic field. The intensification in graphene Maxwell nanofluid velocity can be seen owing to increasing values of Maxwell parameter $(\beta)$ whereas it gets diminished because of unsteadiness parameter $(A)$ increasing values, as revealed from Figures 5 and 6 . Here, the graphene nanofluid profiles are declined with improving values of unsteadiness parameter and consequently reduces the momentum boundary layer thickness. This behaviour of fluid velocity is due to lessening in fluid yield stress in the regime of boundary layer. Further, it is to be noted from Figures 2-6 that in case of no-slip condition the graphene Maxwell nanofluid velocity is higher than that of slip condition. Figure 7 exhibits that the graphene Maxwell nanofluid fluid temperature is getting improved due to augmentation of radiation parameter $(N r)$. It is because of the physical nature of radiation that it upsurges the conduction influence of fluid and therefore, thermal boundary layer gets more thicken and in turn fluid temperature gets reduced. Figure 8 shows that graphene Maxwell nanofluid temperature is improved due to increase in inclination angle of magnetic field. This tendency divulges improvement in boundary layer thickness and reduction in the rate of heat transfer at the boundary. It is apparent from Figure 9 that by increasing the Eckert number $(E c)$, the graphene Maxwell nanofluid temperature can also be increased. The reason behind this behaviour of graphene Maxwell nanofluid is that Eckert number relates the kinetic energy to enthalpy and the total work is done against viscosity where the kinetic energy is converted into internal energy. Hence, viscous dissipation has a nature to raise temperature of fluid in whole boundary layer regime. Figure 10 presents that thermal slip parameter $\varepsilon$ has reducing influence on the graphene Maxwell fluid temperature. Reason for this tendency of fluid temperature is that due to increment in thermal slip parameter, a reduced amount of heat flows from surface to the fluid and subsequently temperature is reduced. Further, it is to be noted from Figures 7-10 that in case of no-slip condition the graphene Maxwell nanofluid temperature is higher than that of slip condition.

Owing to engineering interest viewpoint, we have also computed the numerical date of coefficient of skin friction and Nusselt number for various values of related flow parameters and have presented in tabular form via Tables 3 and 4 respectively. During the computation, we have chosen radiation parameter $N r=0.2$, Prandtl number $P r=5$, unsteadiness parameter $A=0.1$, thermal slip parameter $\varepsilon=0.1$, Eckert number $E c=0.1$, inclination angle of magnetic field $\gamma=\pi / 3$, magnetic parameter $M=1$, Maxwell parameter $\beta=0.5$, and porosity parameter $K_{1}=0.1$. These mentioned values are considered unchanged during the computation while other varying values are shown in tables. It is apparent from Table 3 that skin friction coefficient is augmented at the stretched sheet under both slip and non-slip conditions due to upsurge in magnetic effect, porosity of nanofluid, inclination angle of magnetic field and unsteadiness parameters while Maxwell parameter has adverse impact on it. Table 4 discloses that heat transfer rate gets enhanced owing to increase in radiation, inclination angle 
of magnetic field and thermal slip parameters whereas it gets reduced due to increase in viscous dissipation and unsteadiness parameters.

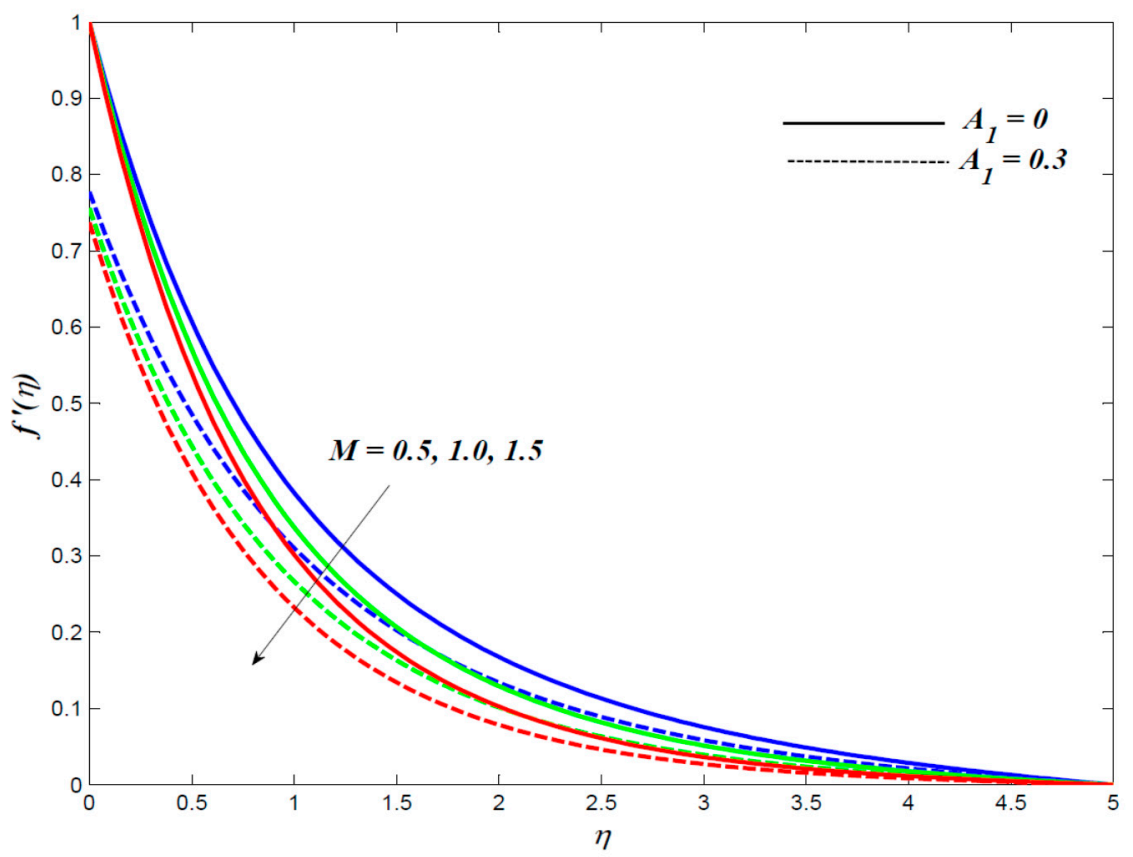

Figure 2. Velocity profiles owing to varying values of $M$.

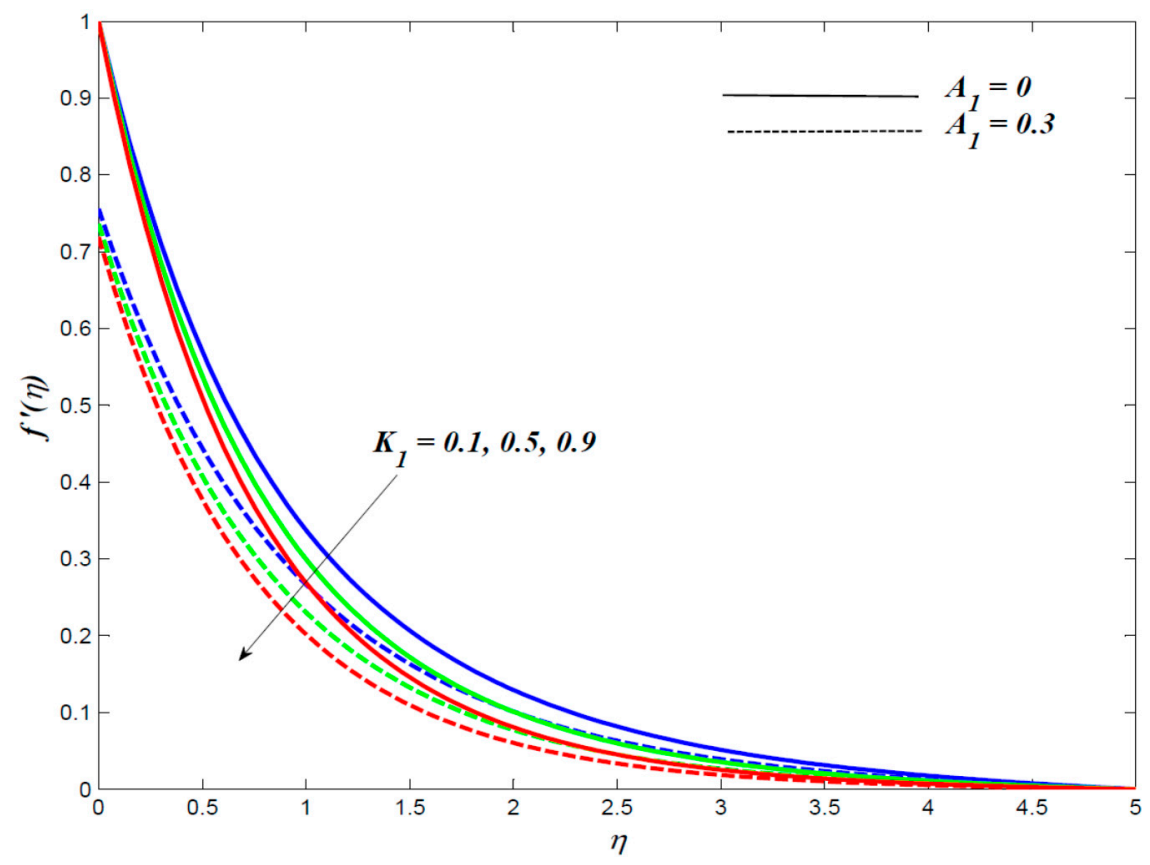

Figure 3. Velocity profiles owing to varying values of $K_{1}$. 




Figure 4. Velocity profiles owing to varying values of $\gamma$.

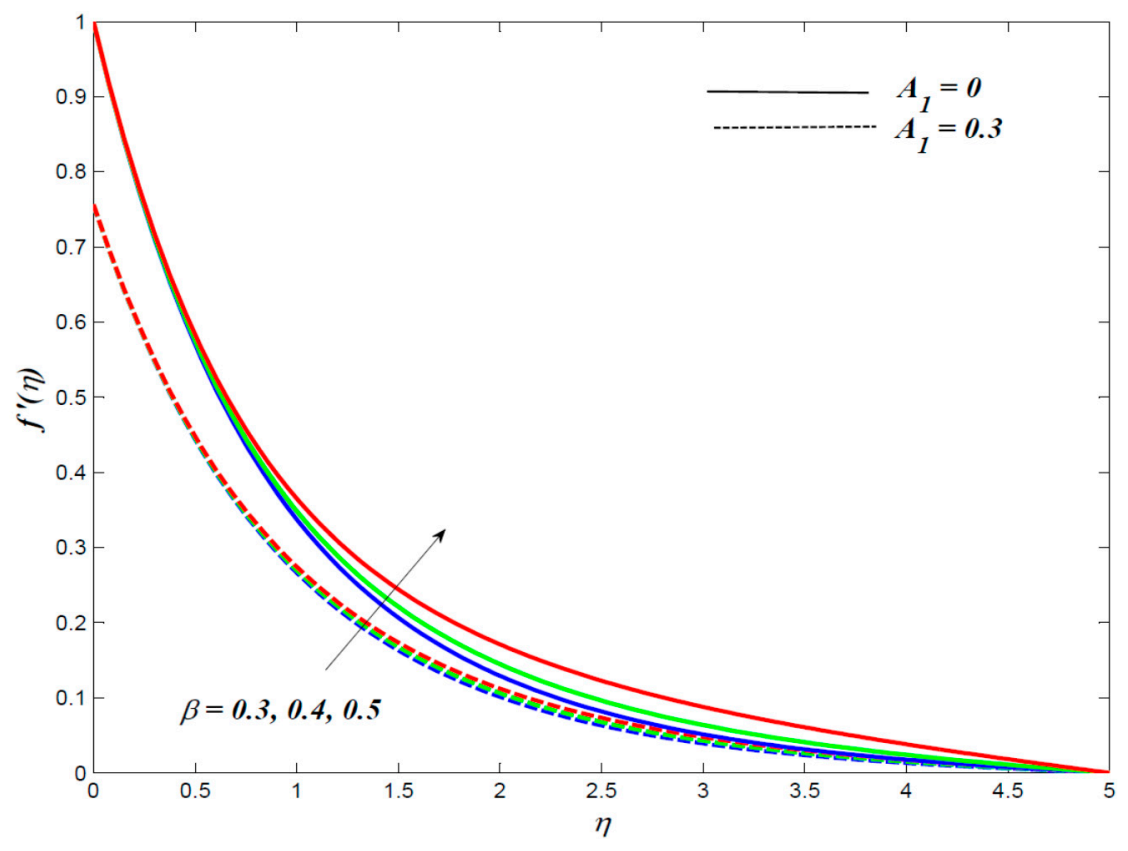

Figure 5. Velocity profiles owing to varying values of $\beta$. 


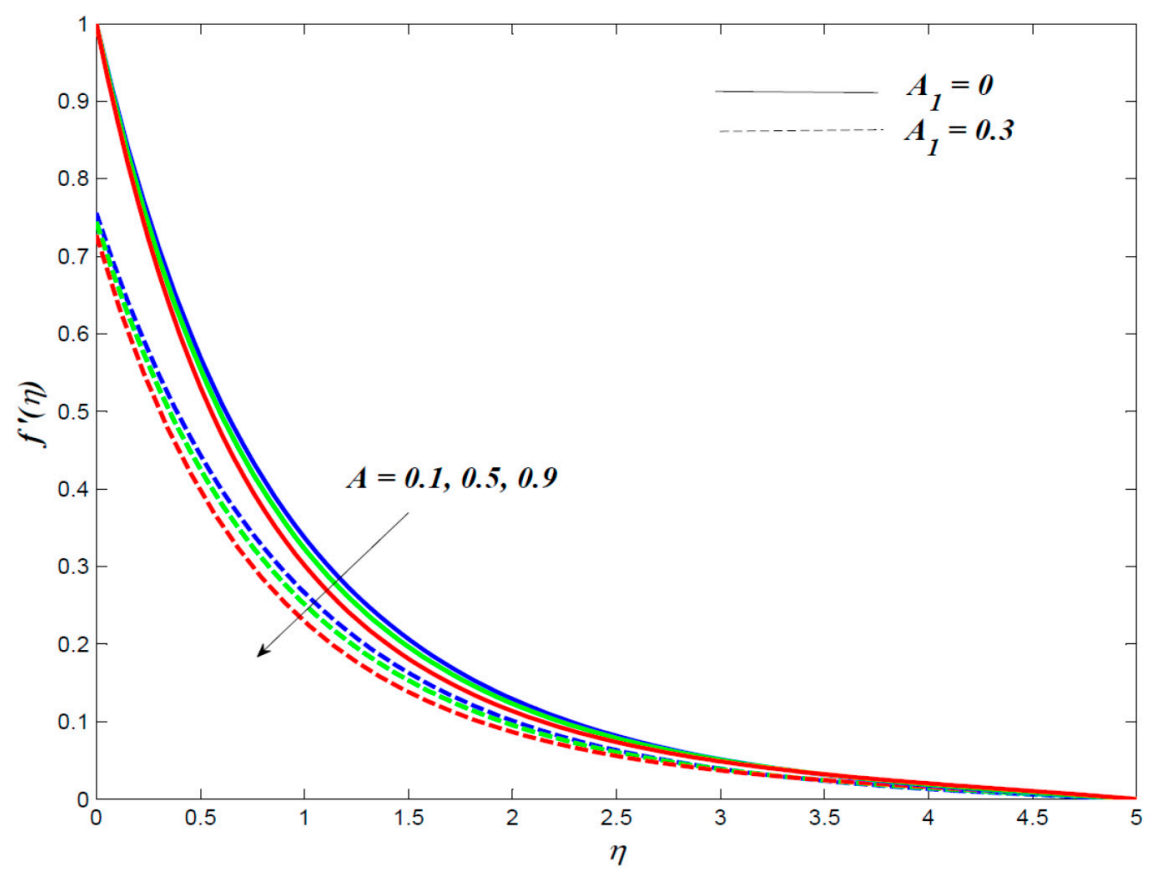

Figure 6. Velocity profile due to variation in unsteadiness parameter.

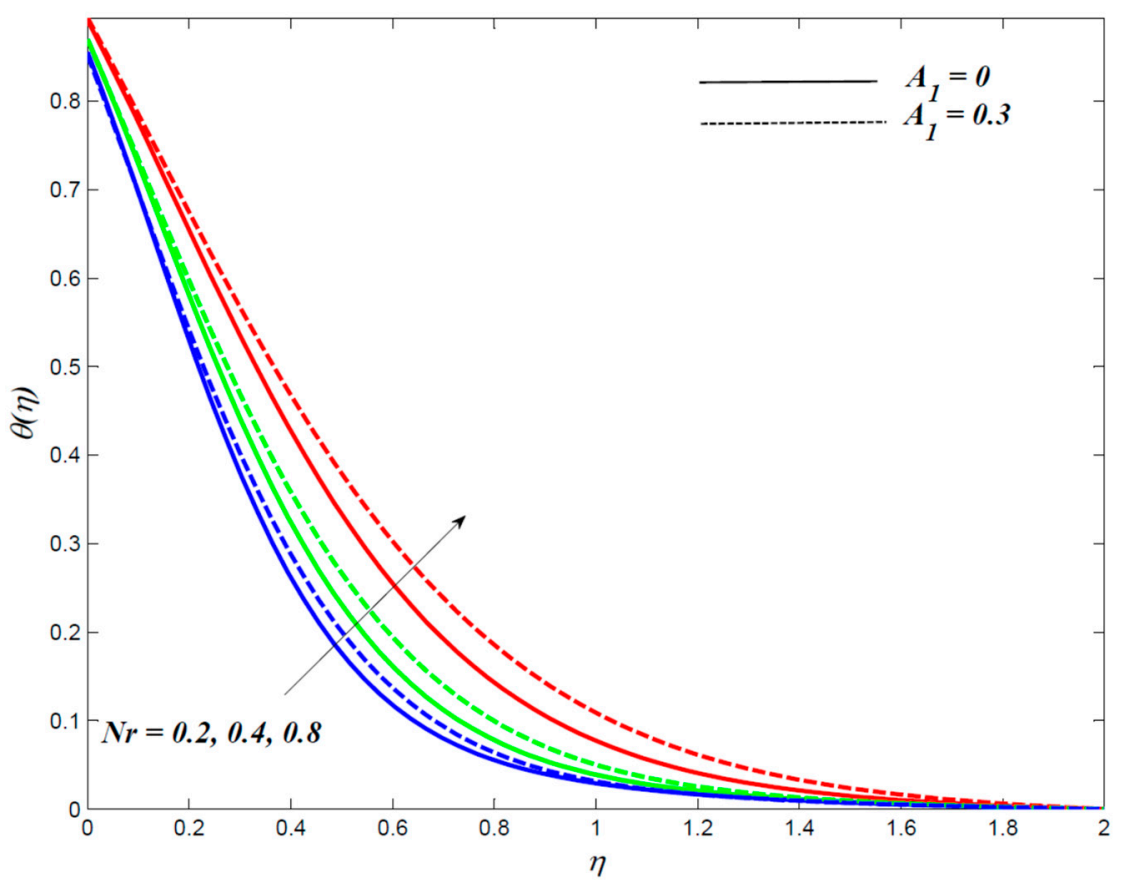

Figure 7. Temperature profiles owing to varying values of $\mathrm{Nr}$. 


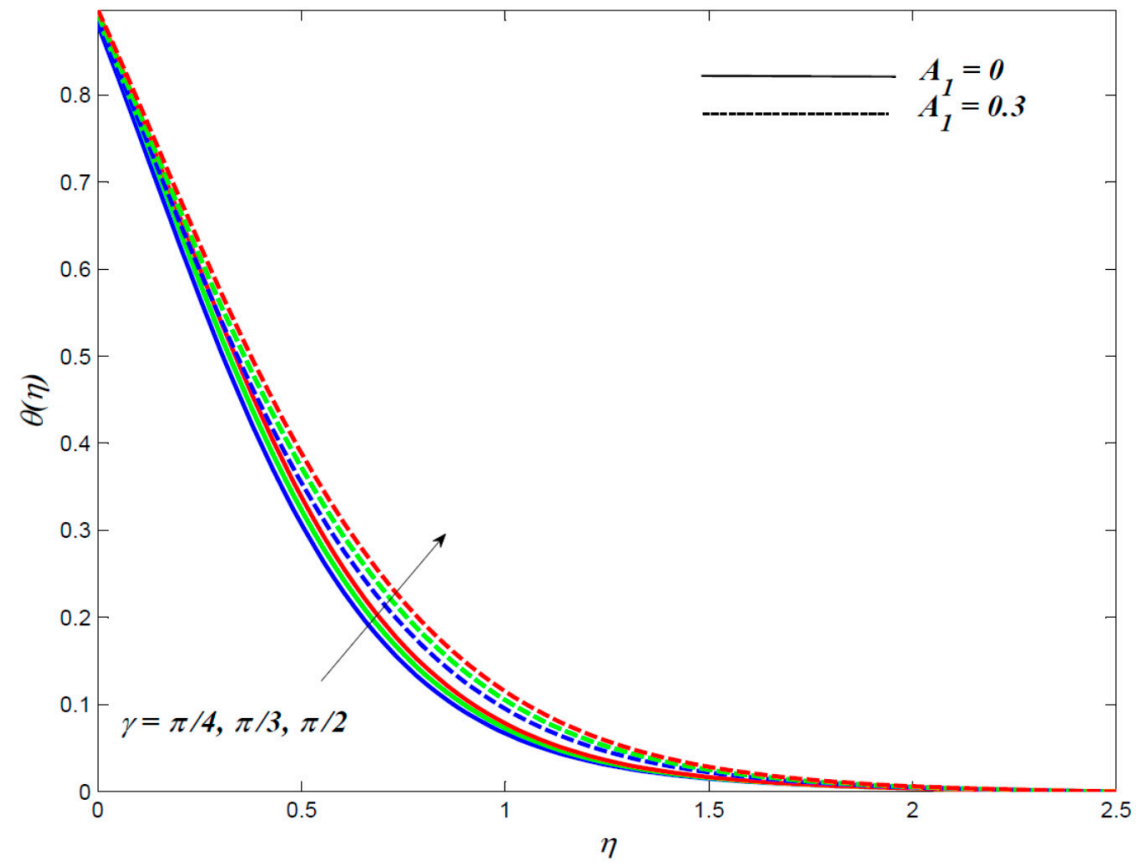

Figure 8. Temperature profiles owing to varying values of $\gamma$.



Figure 9. Temperature profiles owing to varying values of $E c$. 


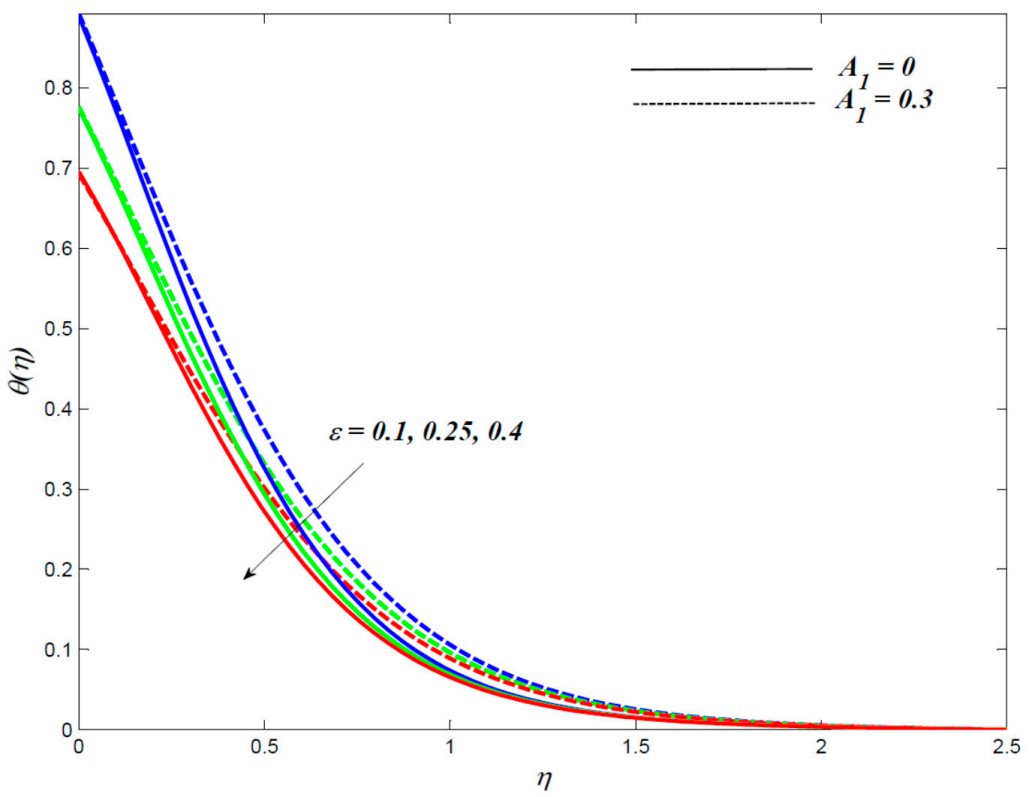

Figure 10. Temperature profiles owing to varying values of $\varepsilon$.

Table 3. Skin friction coefficient under both no-slip $\left(A_{1}=0\right)$ and slip $\left(A_{1}=0.3\right)$ conditions.

\begin{tabular}{|c|c|c|c|c|c|c|}
\hline \multirow{2}{*}{$M$} & \multirow{2}{*}{$K_{1}$} & \multirow{2}{*}{$\gamma$} & \multirow{2}{*}{$\beta$} & \multirow{2}{*}{$A$} & \multicolumn{2}{|c|}{$-S_{f}$ (Skin Friction Coefficient) } \\
\hline & & & & & $A_{1}=0$ & $A_{1}=0.3$ \\
\hline 0.5 & 0.1 & $\pi / 3$ & 0.5 & 0.1 & 0.787368 & 0.570581 \\
\hline 1.0 & & & & & 0.882124 & 0.625921 \\
\hline \multirow[t]{13}{*}{1.5} & & & & & 0.967012 & 0.673289 \\
\hline & 0.1 & & & & 0.882131 & 0.625924 \\
\hline & 0.5 & & & & 0.972382 & 0.676269 \\
\hline & 0.9 & & & & 1.054564 & 0.719928 \\
\hline & & $\pi / 4$ & & & 0.820281 & 0.590109 \\
\hline & & $\pi / 3$ & & & 0.882134 & 0.625925 \\
\hline & & $\pi / 2$ & & & 0.939652 & 0.658243 \\
\hline & & & 0.3 & & 0.882143 & 0.625921 \\
\hline & & & 0.4 & & 0.876278 & 0.624838 \\
\hline & & & 0.5 & & 0.866132 & 0.623478 \\
\hline & & & & 0.1 & 0.889234 & 0.629462 \\
\hline & & & & 0.5 & 0.955132 & 0.666434 \\
\hline & & & & 0.9 & 1.017879 & 0.700464 \\
\hline
\end{tabular}

Table 4. Heat transfer rate at the stretched sheet under both no-slip $\left(A_{1}=0\right)$ and slip $\left(A_{1}=0.3\right)$ conditions.

\begin{tabular}{|c|c|c|c|c|c|c|}
\hline \multirow{2}{*}{$N r$} & \multirow{2}{*}{$\gamma$} & \multirow{2}{*}{$E c$} & \multirow{2}{*}{$\varepsilon$} & \multirow{2}{*}{$A$} & \multicolumn{2}{|c|}{$-N u$ (Nusselt Number) } \\
\hline & & & & & $A_{1}=0$ & $A_{1}=0.3$ \\
\hline 0.1 & $\pi / 3$ & 0.1 & 0.1 & 0.1 & 1.793821 & 1.782463 \\
\hline 0.3 & & & & & 1.609543 & 1.569712 \\
\hline \multirow[t]{13}{*}{0.5} & & & & & 1.352834 & 1.286441 \\
\hline & $\pi / 4$ & & & & 1.595722 & 1.519971 \\
\hline & $\pi / 3$ & & & & 1.493398 & 1.439751 \\
\hline & $\pi / 2$ & & & & 1.396055 & 1.366131 \\
\hline & & 0.10 & & & 1.493372 & 1.439730 \\
\hline & & 0.25 & & & 0.680023 & 0.656432 \\
\hline & & 0.40 & & & 0.580083 & 0.473145 \\
\hline & & & 0.10 & & 1.493371 & 1.439731 \\
\hline & & & 0.25 & & 1.215083 & 1.201544 \\
\hline & & & 0.40 & & 1.024223 & 1.030946 \\
\hline & & & & 0.1 & 1.493362 & 1.439734 \\
\hline & & & & 0.4 & 1.341263 & 1.253533 \\
\hline & & & & 0.7 & 1.157033 & 0.995855 \\
\hline
\end{tabular}




\section{Multiple Regression Analysis: Estimation of Nusselt Number and Skin Friction Coefficients}

In this section, we have used a statistical method for the multiple quadratic regression analysis to estimate the relationship between two or more variables. Generally, regression analysis is useful to know how a precise value of a dependent variable behaves because of the modification of an independent variable while other independent variables are kept fixed. Here, quadratic regression estimation analysis for skin friction coefficients and Nusselt number are reported. The model of multiple quadratic regression estimation for coefficient of skin friction is mentioned for 125 different values of $M$ and $K_{1}$, obtained arbitrarily from intervals [1,7] and [0.1,0.5] respectively for two different values of $\beta=0.1$ and $\beta=0.5$. Apart from this, a model of multiple quadratic regression estimation for Nusselt number is provided for 125 different values of $E c$ and $N r$, analyzed arbitrarily from intervals $[0.1,0.3]$ and $[0.2,0.6]$ for two different values of $\mathrm{Pr}=5$ and $\mathrm{Pr}=10$. During the estimation process, other parameters are considered constant as stated in above Section 4.

The estimated quadratic regression model for $C f_{x} R e_{x}^{1 / 2}$ corresponding to $M$ and $K_{1}$ is given as follows:

$$
C f_{e s t}=C f+b_{1} M+b_{2} K_{1}+b_{3} M^{2}+b_{4} K_{1}^{2}+b_{5} M K_{1} .
$$

While the regression formula for $N u R e_{x}^{1 / 2}$ corresponding to Eckert number Ec and thermal radiation parameter $\mathrm{Nr}$ is given as

$$
N u_{e s t}=N u+c_{1} E c+c_{2} N r+c_{3}(E c)^{2}+c_{4}(N r)^{2}+c_{5} E c N r .
$$

Tables 5 and 6 present the coefficients of multiple quadratic regression estimated values of skin friction coefficients and Nusselt number corresponding to different parameters. The maximum relative error bound for skin friction i.e., $\varepsilon=\left|C f_{\text {est }}-C f\right| / C f$ and for Nusselt number i.e., $\varepsilon_{1}=\left|N u_{\text {est }}-N u\right| / N u$ are also obtained and presented in Tables 5 and 6 , respectively. From the tabulated values, we noticed that the coefficient of permeability of porous medium parameter is higher than that of magnetic field parameter. This suggests that the variation in skin friction coefficient is more sensitive to permeability parameter $K_{1}$ than that of magnetic field parameter $M$ for both values of $\beta$. Correspondingly, we can observe that the Nusselt number is more prone to radiation parameter $N r$ as compared to Eckert number $E c$.

Table 5. The values of error bound $\varepsilon$ and coefficients of multiple quadratic regression for estimated $C f_{x} R e_{x}^{1 / 2}$ due to variations in $M$ and $K_{1}$ are obtained as.

\begin{tabular}{cccccccc}
\hline $\boldsymbol{\beta}$ & $\boldsymbol{C f}$ & $\boldsymbol{b}_{1}$ & $\boldsymbol{b}_{2}$ & $\boldsymbol{b}_{3}$ & $\boldsymbol{b}_{4}$ & $\boldsymbol{b}_{5}$ & $\boldsymbol{\varepsilon}$ \\
\hline 0.1 & -0.9221 & -0.2183 & -0.3291 & 0.0072 & 0.0373 & 0.0228 & 0.0057 \\
0.5 & -0.9154 & -0.2181 & -0.3192 & 0.0069 & 0.0475 & 0.0182 & 0.0062 \\
\hline
\end{tabular}

Table 6. The values of error bound $\varepsilon$ and coefficients of multiple quadratic regression for estimated $N u \operatorname{Re}_{x}^{1 / 2}$ due to variations in $E c$ and $\mathrm{Nr}$ are analyzed as.

\begin{tabular}{cccccccc}
\hline $\boldsymbol{P r}$ & $\mathrm{Nu}$ & $c_{1}$ & $c_{2}$ & $c_{3}$ & $c_{4}$ & $c_{5}$ & $\varepsilon_{1}$ \\
\hline 5 & -3.0414 & 1.2812 & -2.6633 & -0.1794 & 1.0632 & 1.1211 & 0.000001061 \\
10 & -4.0872 & 1.9613 & -4.0411 & 0.0701 & 2.0577 & 1.5837 & 0.000026912 \\
\hline
\end{tabular}

\section{Conclusions}

Owing to noteworthy applications in electronics, energy sector, sensing outlets and medical sciences, in this study, we have investigated the significance of thermal radiation and viscous dissipations on the flow of hydromagnetic graphene Maxwell nanofluid past a linearly horizontal stretched sheet taking momentum and thermal slip conditions into account. Some significant conclusions of the study are summarized as follows: 
- The graphene Maxwell nanofluid velocity gets reduced owing to enhancement in magnetic field, inclination angle of magnetic field, porosity and unsteadiness parameters whereas behaviour of fluid velocity gets reversed due to Maxwell parameter.

- The slip parameter, thermal radiation and viscous dissipation play a significant role to control the temperature of nanofluid.

- The shear stress at the stretched sheet is more sensitive to permeability of the porous medium as compared to magnetic field effect whereas rate of heat transfer is more prone to thermal radiation parameter as compared to viscous dissipation.

- By controlling the thermal radiation, unsteadiness and thermal slip parameters the heat transfer rate can be maintained, which is very useful in manufacturing industries.

Author Contributions: Conceptualization, S.M.H., R.S. and M.R.M.; Methodology, S.M.H. and R.S.; Software, S.M.H. and R.S.; Investigation, S.M.H., R.S., M.R.M. and S.S.A.; Writing-original draft preparation, S.M.H., R.S. and M.R.M. All authors have read and agreed to the published version of the manuscript.

Funding: The two authors Syed M. Hussain and Sattam S. Alrashidy of the paper are thankful to Deanship of Scientific Research, Islamic University of Madinah, Ministry of Education, KSA for financial help under research grant Takamul/11/130.

Acknowledgments: Authors are grateful to the reviewers for their valuable comments and suggestions which helped us to improve the quality of the research paper.

Conflicts of Interest: The authors of the paper have no conflict of interest concerning this publication.

\section{References}

1. Choi, S.U.S.; Eastman, J.A. Enhancing thermal conductivity of fluids with nanoparticles, developments and applications of non-Newtonian flows. In Proceedings of the 1995 International Mechanical Engineering Congress and Exhibition, San Francisco, CA, USA, 12-17 November 1995; Volume 66, pp. 99-105.

2. Eastman, J.A.; Choi, S.U.S.; Li, S.; Yu, W.; Thompson, L.J. Anomalously increased effective thermal conductivities of ethylene glycol-based nanofluids containing copper nanoparticles. Appl. Phys. Lett. 2001, 78, 718-720. [CrossRef]

3. Mahendran, V.; Philip, J. Nanofluid based optical sensor for rapid visual inspection of defects in ferromagnetic materials. Appl. Phys. Lett. 2012, 100, 073104. [CrossRef]

4. Mahendran, V.; Philip, J. An optical technique for fast and ultrasensitive detection of ammonia using magnetic nanofluids. Appl. Phys. Lett. 2013, 102, 063107. [CrossRef]

5. Netro, A.H.C.; Guinea, F.; Peres, N.M.R.; Novoselov, K.S.; Geim, A.K. The electronic properties of grapheme. Rev. Mod. Phys. 2009, 81, 109-162. [CrossRef]

6. Chung, A.; Kim, Y.K.; Shin, D.; Ryoo, S.R.; Hong, B.H.; Min, D.H. Biomedical applications of graphene and graphene oxide. Acc. Chem. Res. 2013, 46, 2211-2224. [CrossRef] [PubMed]

7. Upadhya, M.S.; Mahesha; Raju, C.S.K. Unsteady flow of Carreau fluid in a suspension of dust and graphene nanoparticles with Cattaneo-Christov heat flux. J. Heat Transf. 2018, 140, 092401. [CrossRef]

8. Serdar, B.; Dokuz, M.S. Three-dimensional stagnation point flow of a second-grade fluid towards a moving plate. Int. J. Eng. Sci. 2006, 44, 49-58.

9. Haroun, M.H. Effect of Deborah number and phase difference on peristaltic transport of a third-order fluid in an asymmetric channel. Comm. Nonlinear Sci. Num. Simul. 2007, 12, 464-1480. [CrossRef]

10. Sajid, M.; Hayat, T.; Asgharm, S. Non-similar analytic solution for MHD flow and heat transfer in a third-order fluid over a stretching sheet. Int. J. Heat Mass Transf. 2007, 50, 1723-1736. [CrossRef]

11. Hayat, T.; Noreen, S.; Sajid, M. Heat transfer analysis of the steady flow of a fourth-grade fluid. Int. J. Therm. Sci. 2008, 47, 591-599. [CrossRef]

12. Hayat, T.; Siddiqui, A.M.; Asghar, S. Some simple flows of an Oldroyd-B fluid. Int. J. Eng. Sci. 2001, 39, 135-147. [CrossRef]

13. Fetecau, C.; Fetecau, C. A new exact solution for the flow of Maxwell fluid past an infinite plate. Int. J. Nonlinear Mech. 2003, 38, 423-427. [CrossRef]

14. Wang, Y.; Hayat, T. Fluctuating flow of Maxwell fluid past a porous plate with variable suction. Nonlinear Anal. Real World Appl. 2008, 9, 1269-1282. [CrossRef] 
15. Fetecau, C.; Athar, M.; Fetecau, C. Unsteady flow of a generalized Maxwell fluid with fractional derivative due to a constantly accelerating plate. Comp. Math. Appl. 2009, 57, 596-603. [CrossRef]

16. Hayat, T.; Abbas, Z.; Sajid, M. MHD stagnation point flow of an upper-convected Maxwell fluid over a stretching surface. Chaos Solitons Fractals 2009, 39, 840-849. [CrossRef]

17. Heyhyat, M.; Khabazi, N. Non-isothermal flow of Maxwell fluids above fixed flat plates under the influence of a transverse magnetic field. J. Mech. Eng. Sci. 2011, 225, 909-916. [CrossRef]

18. Wang, J.; Khan, M.I.; Khan, W.A.; Abbas, S.Z.; Khan, M.I. Transportation of heat generation/absorption and radiative heat flux in homogeneous-heterogeneous catalytic reactions of non-Newtonian fluid (Oldroyd-B model). Comput. Methods Prog. Biomed. 2020, 189, 105310. [CrossRef]

19. Nayak, M.K.; Shaw, S.; Khan, M.I.; Pandey, V.S.; Nazeer, M. Flow and thermal analysis on Darcy-Forchheimer flow of copper-water nanofluid due to a rotating disk: A static and dynamic approach. J. Mater. Res. Technol. 2020, 9, 7387-7408. [CrossRef]

20. Khan, M.I.; Alzahrani, F. Activation energy and binary chemical reaction effect in nonlinear thermal radiative stagnation point flow of Walter-B nanofluid: Numerical computations. Int. J. Mod. Phys. B 2020, 34, 2050132. [CrossRef]

21. Hayat, T.; Khan, M.I.; Farooq, M.; Alsaedi, A.; Waqas, M.; Yasmeen, T. Impact of Cattaneo-Christov heat flux model in flow of variable thermal conductivity fluid over a variable thicked surface. Int. J. Heat Mass Transf. 2016, 99, 702-710. [CrossRef]

22. Hussain, S.M.; Jain, J.; Seth, G.S.; Rashidi, M.M. Free convective heat transfer with Hall effects, heat absorption and chemical reaction over an accelerated moving plate in a rotating system. J. Magn. Magn. Mater. 2017, 422, 112-123. [CrossRef]

23. Hussain, S.M.; Jain, J.; Seth, G.S.; Rashidi, M.M. Effect of thermal radiation on magneto-nanofluids free convective flow over an accelerated moving ramped temperature plate. Sci. Iran. B 2018, 25, 1243-1257.

24. Kumar, B.; Seth, G.S.; Nandkeolyar, R. Regression model and successive linearization approach to analyse stagnation point micropolar nanofluid flow over a stretching sheet in a porous medium with nonlinear thermal radiation. Phys. Scr. 2019, 94, 115211. [CrossRef]

25. Ghaffari, A.; Mustafa, I.; Javed, T. Influence of nonlinear radiation on natural convection flow of carbon nanotubes suspended in water-based fluid along a vertical wavy surface. Phys. Scr. 2019, 94, 115214. [CrossRef]

26. Shahzad, M.; Sun, H.; Sultan, F.; Khan, W.A.; Ali, M.; Irfan, M. Transport of radiative heat transfer in dissipative Cross nanofluid flow with entropy generation and activation energy. Phys. Scr. 2019, 94, 115224. [CrossRef]

27. Waqas, H.; Khan, S.U.; Imran, M.; Bhatti, M.M. Thermally developed Falkner-Skan bioconvection flow of a magnetized nanofluid in the presence of a motile gyrotactic microorganism: Buongiorno's nanofluid model. Phys. Scr. 2019, 94, 115304. [CrossRef]

28. Loghmani, G.B.; Heydari, M.; Hosseini, E.; Rashidi, M.M. A numerical simulation of MHD ow and radiation heat transfer of nano uids through a porous medium with variable surface heat $u x$ and chemical reaction. J. Math. Ext. 2019, 13, 31-67.

29. Vajravelu, K.; Hadjinicolaou, A. Heat transfer in a viscous fluid over a stretching sheet with viscous dissipation and internal heat generation. Int. Comm. Heat Mass Transf. 1993, 20, 417-430. [CrossRef]

30. Partha, K.; Murthy, P.V.S.N.; Rajasekhar, G.P. Effect of viscous dissipation on the mixed convection heat transfer from an exponentially stretching surface. Heat Mass Transf. 2005, 41, 360-366. [CrossRef]

31. Cortell, R. Effects of viscous dissipation and radiation on thermal boundary layer over a nonlinearly stretching sheet. Phys. Lett. 2008, 372, 631-636. [CrossRef]

32. Aziz, M.A. Viscous dissipation effect on mixed convection flow of a micropolar fluid over an exponentially stretching sheet. Can. J. Phys. 2009, 87, 359-368. [CrossRef]

33. Daniel, Y.S.; Aziz, Z.A.; Ismail, Z.; Salah, F. Effects of thermal radiation, viscous and Joule heating on electrical MHD nanofluid with double stratification. Chin. J. Phys. 2017, 55, 630-651. [CrossRef]

34. Seth, G.S.; Singh, J.K. Mixed convection hydromagnetic flow in a rotating channel with Hall and wall conductance effects. Appl. Math. Mod. 2016, 40, 2783-2803. [CrossRef]

35. Seth, G.S.; Sarkar, S.; Makinde, O.D. Combined free and forced convection Couette-Hartmann flow in a rotating channel with arbitrary conducting walls and Hall effects. J. Mech. 2016, 32, 613-629. [CrossRef]

36. Abbas, S.Z.; Khan, W.A.; Kadry, S.; Khan, M.I.; Waqas, M.; Khan, M.I. Entropy optimized Darcy-Forchheimer nanofluid (Silicon dioxide, Molybdenum disulfide) subject to temperature dependent viscosity. Comput. Methods Prog. Biomed. 2020, 190, 105363. [CrossRef] [PubMed] 
37. Wang, J.; Muhammad, R.; Khan, M.I.; Khan, W.A.; Abbas, S.Z. Entropy optimized MHD nanomaterial flow subject to variable thicked surface. Comput. Methods Prog. Biomed. 2020, 189, 105311. [CrossRef] [PubMed]

38. Nayak, M.K.; Hakeem, A.K.A.; Ganga, B.; Khan, M.I.; Waqas, M.; Makinde, O.D. Entropy optimized MHD 3D nanomaterial of non-Newtonian fluid: A combined approach to good absorber of solar energy and intensification of heat transport. Comput. Methods Prog. Biomed. 2020, 186, 105131. [CrossRef]

39. Ibrahim, M.; Khan, M.I. Mathematical modeling and analysis of SWCNT-Water and MWCNT-Water flow over a stretchable sheet. Comput. Methods Prog. Biomed. 2020, 187, 105222. [CrossRef]

40. Mukhopadhyay, S.; Gorla, R.S.R. Effects of partial slip on boundary layer flow past a permeable exponential stretching sheet in presence of thermal radiation. Heat Mass Transf. 2012, 48, 1773-1781. [CrossRef]

41. Singh, G.; Makinde, O.D. Mixed convection slip flow with temperature jump along a moving plate in presence of free stream. Therm. Sci. 2015, 19, 119-128. [CrossRef]

42. Seth, G.S.; Sharma, R.; Mishra, M.K.; Chamkha, A.J. Analysis of hydromagnetic natural convection radiative flow of a viscoelastic nanofluid over a stretching sheet with Soret and Dufour effects. Eng. Comp. 2017, 34, 603-628. [CrossRef]

43. Seth, G.S.; Tripathi, R.; Mishra, M.K. Hydromagnetic thin film flow of Casson fluid in non-Darcy porous medium with Joule dissipation and Navier's partial slip. Appl. Math. Mech. 2017, 38, 1613-1626. [CrossRef]

44. Khan, M.I.; Alzahrani, F.; Hobiny, A. Simulation and modeling of second order velocity slip flow of micropolar ferrofluid with Darcy-Forchheimer porous medium. J. Mater. Res. Technol. 2020, 9, 7335-7340. [CrossRef]

45. Abbas, S.Z.; Khan, M.I.; Kadry, S.; Khan, W.A.; Israr-Ur-Rehman, M.; Waqas, M. Fully developed entropy optimized second order velocity slip MHD nanofluid flow with activation energy. Comput. Methods Prog. Biomed. 2020, 190, 105362. [CrossRef] [PubMed]

46. Khan, M.I.; Alzahrani, F.; Hobiny, A. Heat transport and nonlinear mixed convective nanomaterial slip flow of Walter-B fluid containing gyrotactic microorganisms. Alex. Eng. J. 2020, 59, 1761-1769. [CrossRef]

47. Muhammad, R.; Khan, M.I.; Khan, N.B.; Jameel, M. Magnetohydrodynamics (MHD) radiated nanomaterial viscous material flow by a curved surface with second order slip and entropy generation. Comput. Methods Prog. Biomed. 2020, 189, 105294. [CrossRef]

48. Muhammad, R.; Khan, M.I.; Jameel, M.; Khan, N.B. Fully developed Darcy-Forchheimer mixed convective flow over a curved surface with activation energy and entropy generation. Comput. Methods Prog. Biomed. 2020, 188, 105298. [CrossRef]

49. Khan, M.I.; Alzahrani, F.; Hobiny, A.; Ali, Z. Modeling of Cattaneo-Christov double diffusions (CCDD) in Williamson nanomaterial slip flow subject to porous medium. J. Mater. Res. Technol. 2020, 9, 6172-6177. [CrossRef]

50. Khan, M.I.; Qayyum, S.; Kadry, S.; Khan, W.A.; Abbas, S.Z. Irreversibility analysis and heat transport in squeezing nanoliquid flow of non-Newtonian (second-grade) fluid between infinite plates with activation energy. Arab. J. Sci. Eng. 2020, 45, 4939-4947. [CrossRef]

51. Aziz, A.; Jamshed, W.; Ali, Y.; Shams, M. Heat transfer and entropy analysis of Maxwell hybrid nanofluid including effects of inclined magnetic field, Joule heating and thermal radiation. Dis. Contin. Dyna. Syst.-S 2020, 13, 2667-2690. [CrossRef]

52. Babu, M.J.; Sandeep, N. 3D MHD slip flow of a nanofluid over a slendering stretching sheet with thermophoresis and Brownian motion effects. J. Mol. Liquids 2016, 222, 1003-1009. [CrossRef]

53. Cramer, R.; Pai, S.I. Magneto Fluid Dynamics for Engineers and Applied Physicist; McGraw-Hill Book Company: Washington, DC, USA, 1973.

54. Mukhopadhyay, S. Heat transfer analysis of the unsteady flow of a Maxwell fluid over a stretching surface in the presence of a heat source/sink. Chin. Phys. Lett. 2012, 29, 054703. [CrossRef]

55. Brewster, M.Q. Thermal Radiative Transfer and Properties; John Wiley and Sons: Hoboken, NJ, USA, 1992.

56. Elbashbeshy, E.M.A.; Bazid, M.A.A. Heat transfer over an unsteady stretching surface with internal heat generation. Appl. Math. Comp. 2003, 138, 239-245. [CrossRef]

Publisher's Note: MDPI stays neutral with regard to jurisdictional claims in published maps and institutional affiliations. 